\title{
Evaluation of periodic contributors to climatic temperature fluctuations from historic data
}

\author{
E.F. Jones and P.M. Gore \\ FM Research Professionals, PO Box 58412, Nashville, TN 37205, USA
}

\begin{abstract}
The purpose of this study is to fit the record of mean global temperature anomalies by using a logarithmic function of the atmospheric carbon dioxide concentration and to determine any periodic contributors to the temperature anomaly record.
\end{abstract}

\section{Mean temperature anomalies}

Parametric fitting of the historical global mean temperature anomalies [1] was performed. A logarithmic function of $\mathrm{CO}_{2}$ concentration was the best fit to the historical data.

The fit of mean global temperature anomaly vs. atmospheric $\mathrm{CO}_{2}$ concentration yielded the equation

$$
\Delta \mathrm{T}=\left(15.999+2.7550 \ln \left(\mathrm{C}_{\mathrm{CO}_{2}}\right)\right) \mathrm{C}^{\circ}
$$

with a coefficient of determination $\mathrm{r}^{2}=0.79068$. A logarithmic dependence of the mean temperature anomaly on $\mathrm{CO}_{2}$ concentration would result if the primary effect of increased $\mathrm{CO}_{2}$ concentration is to raise the height to which the atmosphere is relatively opaque to the absorbed bands of infrared radiation from the Earth's surface [2] and thus the temperature is directly proportional to the change $\Delta \mathrm{F}$ in radiative forcing as a function of changing concentration for carbon dioxide is given by

$$
\Delta F=5.35 \times \ln \frac{C}{C_{0}} W m^{-z}
$$

where $\mathrm{C}$ is the $\mathrm{CO}_{2}$ concentration in parts per million by volume and $\mathrm{C}_{0}$ is the reference concentration [3]. The radiative forcing due to increased atmospheric $\mathrm{CO}_{2}$ is proportional to the log of the $\mathrm{CO}_{2}$ concentration. The increase in mean global temperature anomaly due to the increased $\mathrm{CO}_{2}$ concentration is linearly proportional to the radiative forcing and thus proportional to the log of the $\mathrm{CO}_{2}$ concentration.

\section{Sinusoidal fits}

Adding a sinusoidal function of time with a 21-year period corresponding to the solar cycle improved the fit. Adding another sinusoidal term with a 70-year period further improved the fit.

This could correspond to the Atlantic multidecadal oscillation [4]. The RMS of the errors of the fit from 1850 to 2011 is $0.092 \mathrm{C}^{\circ}$.

This is an Open Access article distributed under the terms of the Creative Commons Attribution License 4.0, which permits unrestricted use, distribution, and reproduction in any medium, provided the original work is properly cited. 


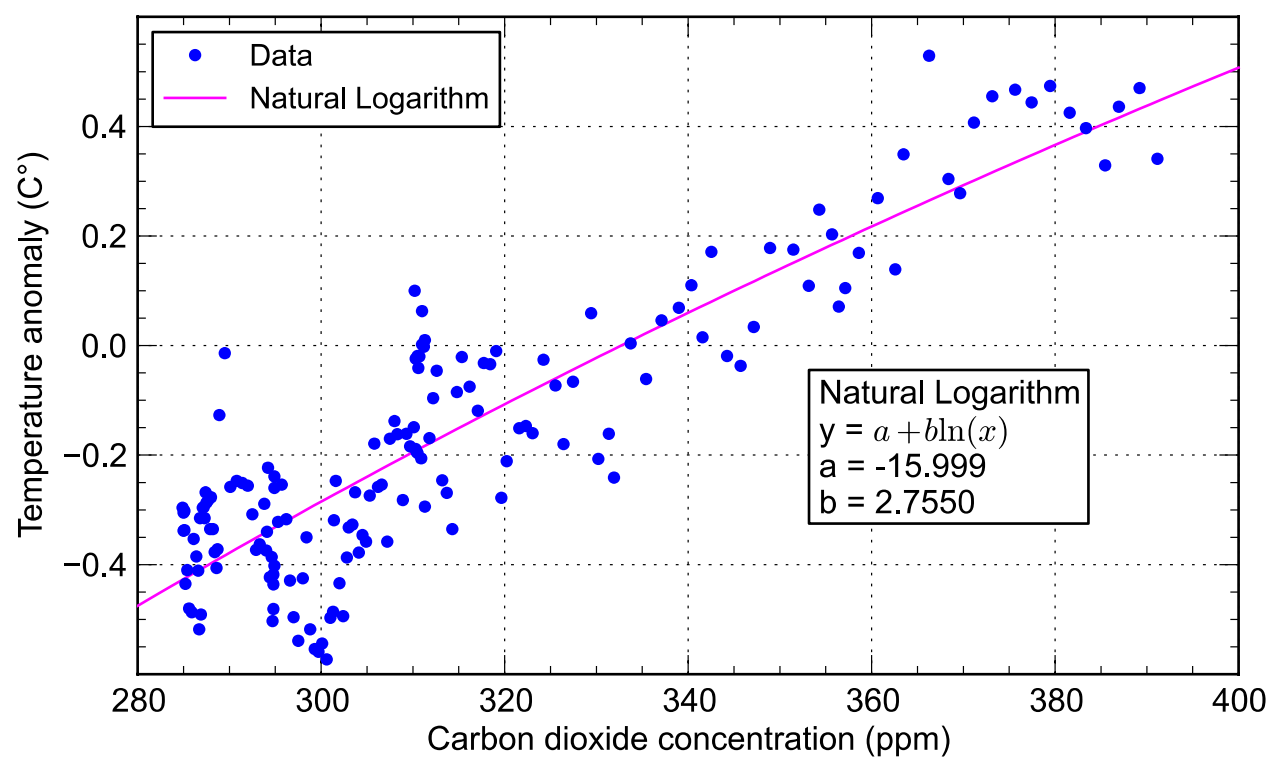

Figure 1. Mean global temperature anomaly vs. atmospheric $\mathrm{CO}_{2}$ concentration.

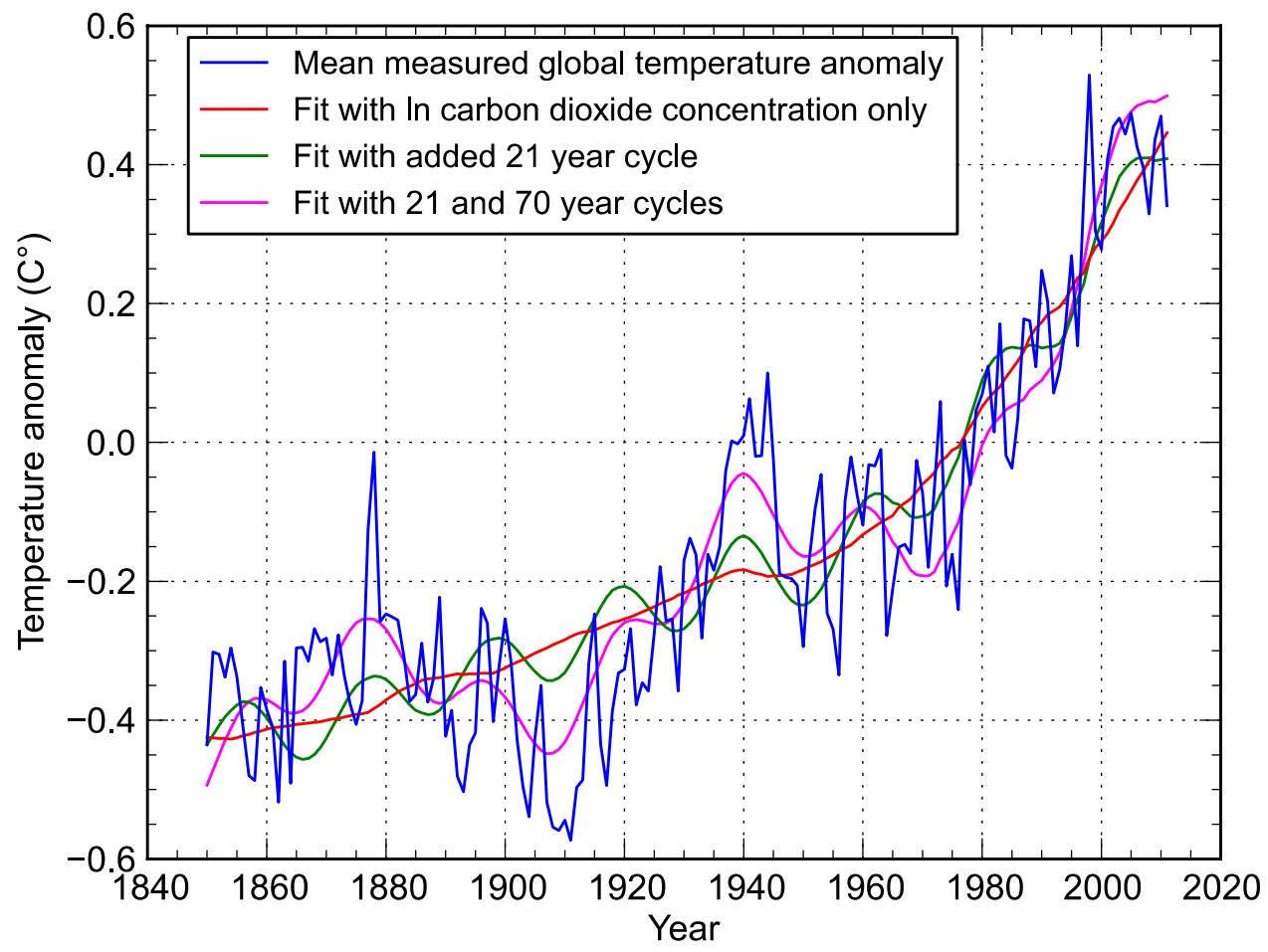

Figure 2. Mean global temperature anomaly vs. year and least squares fits using, respectively, the log of the $\mathrm{CO}_{2}$ concentration, the log of the $\mathrm{CO}_{2}$ concentration plus a 21-year cycle oscillation, and the log of the $\mathrm{CO}_{2}$ concentration plus both 21-year and 70-year oscillations. 


\section{$3^{\text {rd }}$ European Energy Conference}

The formula resulting in the best fit was

$$
\Delta T=\left(2.7712 \ln \frac{C}{333.42}+0.0503 \sin \frac{2 \pi(t-6.393)}{20.957}+0.0981 \sin \frac{2 \pi(t-57.715)}{69.167}\right) \mathrm{C}^{\circ}
$$

where $C$ is the atmospheric carbon dioxide concentration in ppm and $t$ is the calendar year C.E. If we assume that the $\mathrm{CO}_{2}$ linearly increases to $1000 \mathrm{ppm}$ by the year 2100, then we expect the temperature anomaly to be $+3 \mathrm{C}^{\circ}$ in the year 2100 . This greatly simplified analysis ignores positive feedback mechanisms that may occur as the mean temperature anomaly rises.

\section{Summary}

In summary, a logarithmic dependence of the historical mean global temperature anomaly to the atmospheric carbon dioxide concentration yielded a good fit. Adding periodic contributions with 11-year and 70-year cycles further improved the fit. With a linear $\mathrm{CO}_{2}$ increase to $1000 \mathrm{ppm}$ by 2100 , the expectation is $\mathrm{a}+3 \mathrm{C}^{\circ}$ temperature anomaly in 2100 .

\section{References}

[1] P. D. Jones, D. H. Lister, T. J. Osborn, C. Harpham, M. Salmon, C. P. Morice, J. Geophys. Res., 117, D05127 (2012)

[2] S. Manabe, R. T. Wetherald, J. Atmos. Sci., 24, 241 (1967)

[3] T. Augustsson, V. Ramanathan, J. Atmos. Sci., 34, 448 (1977)

[4] M. Dima, G. Lohmann, J. Climate, 20, 2706 (2007) 